\title{
Management of prenatal ovarian cysts
}

Takako Shimada ${ }^{1}$, Kiyonori Miura ${ }^{1}$, Hideo Gotoh ${ }^{2}$, Daisuke Nakayama ${ }^{1}$, Hideaki

$$
\text { Masuzaki }^{1}
$$

${ }^{1}$ Department of Obstetrics and Gynaecology, Nagasaki University Graduate

School of Biomedical Sciences, 1-7-1 Sakamoto, Nagasaki 852-8501, Japan.

${ }^{2}$ Gotoh Obstetrics and Gynecology Hospital,

364 Nishiohmurahon-machi, Ohmura, Japan

2,193 words including abstract and text with two tables and references

Corresponding author:

Takako Shimada, MD,

Department of Obstetrics and Gynecology,

Nagasaki University Graduated School of Biomedical Sciences,

1-7-1 Sakamoto, Nagasaki 852-8501, Japan

Phone: +81-95-849-7363

Fax: +81-95-849-7365

Email: shimachan-ngs@umin.ac.jp 


\begin{abstract}
Objectives: The aim of the present study was to analyze the antenatal and postnatal outcome of fetal ovarian cysts in relation to their ultrasonographic pattern and size. Methods: Sixteen fetal ovarian cysts were diagnosed in 16 fetuses and followed with serial ultrasonograms in utero and after birth until spontaneous or surgical resolution. Results: Eleven fetal ovarian cysts were simple cysts at first prenatal scan but 3 of the 11 became complex cysts at last prenatal scan and required postnatal laparoscopic surgery. Seven of the 11 simple cysts (63\%) disappeared on follow up imaging by ultrasonograms or MRI during pregnancy or within 2 months after birth. The rate of spontaneous resolution of simple cysts was higher than that of complex cysts $(40.0 \%)$. The mean maximum diameter of the ovarian cysts before delivery that were subsequently excised surgically at postnatal period $(50 \pm 13.4 \mathrm{~mm})$ was not different from that of ovarian cysts that resolved spontaneously $(42.8 \pm 12.8 \mathrm{~mm}, \mathrm{P}=0.2918)$.

Conclusion: In our study, cyst size did not predict the risk of ovarian loss. The opportunity of laparoscopic exploration versus conservative management needs to be investigated because some complex cysts resolved spontaneously in the postnatal period.
\end{abstract}




\section{Introduction}

The rate of detection fetal ovarian cysts has increased since the advent of routine antenatal sonography [1]. The etiology of fetal ovarian cysts is not entirely clear. Maturation of the hypothalamus-pituitary-ovary axis commences from the $29^{\text {th }}$ week of gestation under elevated levels of fetoplacental estrogen [2]. An immature hypothalamus-pituitary-ovarian feedback is thought to be responsible for gonadal hyperstimulation in premature fetuses. After delivery, reduction in hormonal stimulation can lead to spontaneous resolution of the ovarian cyst; therefore, a conservative management of uncomplicated cysts has been suggested, although some cysts carry the risk of subsequent complication that might require postnatal surgery [3-5]. Various complications of ovarian cysts have been described, such as compression of neighboring viscera, rupture of the cyst, hemorrhage and ovarian torsion [6-9]. However, there is no standard treatment of fetal ovarian cysts and their management varies widely among different centers. The present retrospective study was conducted to analyze the antenatal and postnatal outcome of fetal ovarian cysts in relation to their ultrasonographic pattern and size.

\section{Materials and Methods}

A total of 16 fetal ovarian cysts were diagnosed in 16 fetuses between 30 and 37 weeks of gestation on routine sonography conducted between 1989 and 2006 in our hospitals. The detection of a cystic structure in the fetal abdomen with otherwise 
normal anatomy of the gastrointestinal and urinary tracts in a female fetus was highly suggestive of an ovarian cyst. This assumption was based on the following fact: other anomalies that can be mistaken as ovarian cysts, such as mesenteric cysts, urachal cysts, enteric duplication, and dilated bowel, are very rare. The shape of enteric bowel duplication is generally tubular, and the difference is a two layered muscle wall seen on antenatal ultrasonography in bowel duplication. But duplications of the gastrointestinal tract are mimick fetal ovarian cysts. Duodenal atresia has a typical double bubble appearance. In cases with a suspected ovarian cyst, a thorough sonographic assessment with/without MR imaging of the entire fetus was performed to exclude other associated anomalies.

The cysts were classified as either complex or simple according to Nussbaum et al. [1]. A cyst that was completely anechoic and had a thin wall was defined as simple, while an echogenic cyst was defined as complex (Figure 1). We measured the maximum antenatal size of the ovarian cyst, and the associated complications and outcome.

After diagnosis, the mothers were followed by serial ultrasound scans every 2 weeks until delivery, and the newborn was followed postnatally until spontaneous resolution or surgical excision. Spontaneous vaginal delivery took place in all cases except when cesarean section was indicated for obstetrical reasons not related to the fetal ovarian cyst. All study protocols were approved by the Committee for Ethical Issues on Human Genome and Analysis of Nagasaki University. 


\section{Results}

The mean diameter of the 16 fetal ovarian cysts was $42.6 \pm 12.3 \mathrm{~mm}( \pm \mathrm{SD})$ at diagnosis. All cysts were first detected in the third trimester. Eleven fetal cysts were simple and five were complex when first detected. All 16 fetuses were born at term, with 14 by normal vaginal delivery and 2 by Cesarean section because of breech presentation or cephalopelvic disproportion.

Spontaneous resolution during pregnancy was noted in 2 of the 11 (18.2\%) simple cysts. Five simple cysts diminished in size 6 months after delivery and 4 of these 5 disappeared spontaneously within 2 months after birth, while surgery was performed for enucleation of one simple cyst due to its large size $(62 \mathrm{~mm})$ at last prenatal diagnosis (Table 1. Case 3). We didn't aspirate for this case because we took into consideration the possibility of fetal ovarian tumor. Histopathological examination of this cyst showed a simple cyst with no specific epithelial findings.

With regard to the remaining 4 (36.4\%) simple cysts, their size did not change or increase at late pregnancy. Three of these simple cysts that did not change in size became the complex type during the prenatal follow-up scans. Repeat sonograms showed a fluid level in the cyst or a solid pattern. Accordingly, surgery was performed within 10 months after birth due to suspected torsion or hemorrhage (Table 1). One of these three cysts showed evidence of torsion at surgery (Table 1, Patient 9) and histopathological examination indicated a necrotic simple cyst with calcification. The other two cysts showed evidence of intracystic hemorrhage but not that of torsion (Table 1, Patients 10 and 11). Histopathological analysis indicated two simple cysts 
with bleeding with or without calcification. One simple cyst that increased in size later showed spontaneous resolution within 6 months after birth (Table 1). What happened to the ovary after the cyst resolved remains unknown, because we did not perform the second look observation by laparoscopy.

With regard to the five complex cysts, four of them showed no change in size during prenatal ultrasound monitoring. Postnatally, three (75\%) of 4 infants underwent surgery for persistence of the cyst or postnatal evidence of torsion or evidence of neoplastic changes (Table 1, Patients 12, 13 and 14). Histopathological examination showed two simple cysts with torsion and one simple cyst with intracystic hemorrhage. The other two complex cysts disappeared on follow up imaging within 11 months after birth as confirmed by follow-up ultrasonography or other imaging. We don't know whether the ovary after the cyst resolved leaved a normal ovary or disappeared on follow up imaging because the laparoscopic second look observation was not performed in all infants.

Surgery was performed for excision of 7 of 16 cysts. The procedure included oophorectomy, sometimes with enucleation. Oophorectomy was performed to the cases that were detected the ischemic necrosis and self-amputation of the fetal ovarian cyst. Microscopic examination indicated that all seven cysts were simple cysts (Table 1). Three out of the seven cysts were simple cysts with torsion, but adhesion between the bowel and ovary was noted in one case during surgery. The other three cysts showed intracystic hemorrhage and one cyst was simple cyst without specific epithelial findings. The histopathological finding was benign and neoplastic tumor was not detected in our study. But Pietro Bagolan et al recommended the surgery of 
the ovarian cysts with an ultrasound pattern of torsion which persisting after birth[8]. We also think that fetal ovarian complex cysts should be managed carefully after birth because 3 of 6 complex cysts that surgeries were performed were cysts with torsion and 1 of 3 cysts with torsion showed the adhesion between the bowel and the cyst.

The mean maximum diameter of the ovarian cysts before delivery that were subsequently excised surgically at postnatal period $(50 \pm 13.4 \mathrm{~mm})$ was not different from that of ovarian cysts that resolved spontaneously (42.8 $\pm 12.8 \mathrm{~mm}, \mathrm{P}=0.2918)$.

\section{Discussion}

The majority of fetal ovarian cysts are considered to result from excessive hormonal stimulation $[10,11]$ and resolve spontaneously after birth and that they are of no clinical significance. The detection of ovarian cysts in utero has increased lately, with the increased use of routine ultrasonography, raising questions on the perinatal management of these anomalies. After the diagnosis of fetal ovarian cyst, serial ultrasound examinations are necessary in order to detect any torsion. It has been suggested that such complication depends on the size of the fetal ovarian cyst [6]. However, there was no such correlation in our series, as we did not detect any difference in the mean size of cysts with and without torsion at diagnosis. The mean maximum diameter of ovarian cysts with torsion was $44.6 \pm 12.1 \mathrm{~mm}$, while that without torsion was $44.2 \pm 12.8 \mathrm{~mm}$. 
Interestingly, 7 of the 11 simple cysts (63.6\%) disappeared spontaneously during pregnancy or within 2 months after birth. The rate of spontaneous resolution of simple cysts was higher than that of complex cysts (40.0\%).

Surgery was performed for excision of seven cysts. Indications for surgeries in our hospital were the following: 1) large fetal ovarian cysts that did not diminish in size at postnatal scan, 2) complex cysts that showed evidence of torsion at postnatal scan, and 3) cysts suspected to be neoplastic tumor. In our experience, cyst size did not predict the risk of ovarian loss; the mean of maximum prenatal diameter of ovarian cysts that were subsequently excised surgically was not different from that of cysts that resolved spontaneously.

We did not carry out any ovarian cyst aspiration during fetal and neonatal life during the study period. Although aspiration of large simple cysts might prevent serious complications, we didn't aspirate them because we took into consideration the possibility of fetal malignant ovarian tumor. And the selection of cases to undergo aspiration is difficult because 7 of the 11 simple cysts (63.6\%) disappeared spontaneously during pregnancy or within 2 months after birth. After the diagnosis of fetal ovarian cyst is established, we recommend a conservative approach that includes serial ultrasound examinations and follow the course of the anomaly until spontaneous resolution.

Complex cysts should be managed carefully because they may represent entities such as intestinal duplications or tumors. Fetal cystadenomas and granulosa cell tumors have been reported in the literature $[14,15]$. It is possible that intracyst hemorrhage 
could result in fetal anemia or torsion of the cyst could cause bowel obstruction [8, $12,13]$.

In our study, two out of eight complex cysts resolved spontaneously. A cyst with an echo pattern of torsion that disappears spontaneously is clinically important, but knowledge of the real nature of such cyst is difficult. Gohar et al, in their report of the case of spontaneously disappearing cyst, suggested that it could be represented by a cyst that underwent initial torsion and subsequent spontaneous detorsion [16]. What happened to the ovary after the cyst resolved remains unknown, because we did not perform the second look observation by laparoscopy, but we also think that there is a possibility of the spontaneous detorsion of the fetal ovarian tumor. Pietro Bagolan et al reported the case that spontaneously disappeared at ultrasound, and said the possibility of the self-amputation of the ovary and adhesion to other organs [8].

Furthermore, laparoscopic surgery was performed to remove four out of seven fetal ovarian cysts. Since the number of cases in this report was small, it is difficult to determine the conditions that dictate a requirement for, and a timing of any surgical intervention. However, from the point of view of minimally invasive management, laparoscopic exploration or conservative management may be necessary as the first line intervention in terms of diagnosis and therapy of the fetal ovarian cysts. 


\section{References}

[1]- Nussbaum AR, Sanders RC, Hartman DS, Dudgeon DL, Parmley TH, Neonatal ovarian cysts; Sonographic-pathologic correlation. Pediatr Radiol 1988; 168: 817-821. [2]- Grumbach MM, Kaplan SL: Fetal pituitary hormones and the maturation of central nervous system regulation of anterior pituitary function, in Gluck L. Modern Perinatal Medicine. Chicago, IL, Year Book, 1975; pp247-256.

[3]- Brandt ML, Luks FI, Filiatrault D, Garel L, Desjardins JG, Youssef S: Surgical indications in antenatally diagnosed ovarian cysts. J Pediatr Surg 1991; 26: 276-281. [4]- Ikeda K, Suita S, Nakano H: Management of ovarian cysts detected antenatally. J Pediatr Surg 1988; 23: 432-435.

[5]- Meizner I, Levy A, Katz M, Maresh AJ, Glezerman M: Fetal ovarian cysts: prenatal ultrasonographic detection and postnatal evaluation and treatment. Am J Obstet Gynecol 1991; 164: 874-878.

[6]- Giorlandino C, Bilancioni E, Bagolan P, Muzii L, Rivosecchi M, Nahom A: Antenatal ultrasonographic diagnosis and management of fetal ovarian cysts. Int $\mathrm{J}$ Gynecol Obstet 1993; 44: 27-31.

[7]- van der Zee DC, van Seumeren IGC, Bax CMA, Rovekamp MH, ter Gunne AJP: Laparoscopic Approach to surgical management of ovarian cysts in the newborn. $\mathrm{J}$ Pediatr Surg 1995; 30: 42-43.

[8]- Bagolan P, Giorlandino C, Nahom A, Bilancioni E, Trucchi A, Gatti C, Aleandri V, Spina V: The management of fetal ovarian cysts. J Pediatr Surg 2002; 37: 25-30. [9]- Foley PT, Ford WDA, McEwing R, Furness M: Is conservative management of prenatal and neonatal ovarian cysts justifiable? Fetal Diagn Ther 2005; 20: 454-458. [10]- Pryse-Davis J, Dewhurst CJ: The development of the ovary and uterus in the foetus, newborn and infant: a morphological and enzyme histochemical study. J Pathol 1971; 103: 5-25.

[11]- deSa DJ: Follicular ovarian cysts in stillbirths and neonates. Arch Dis Child 1975; 50: 45-50.

[12]- Sherer DM, Shah, YG, Eggers, PC, Woods JP Jr: Prenatal sonographic diagnosis and subsequent management of fetal adnexal torsions. J Ultrasound Med 1990; 9: 
161-163,

[13]- Abolmakarem H, Tharmaratnum S, Thilaganathan B: Fetal anaemia as a consequence of haemorrhage into an ovarian cyst. Ultrasound Obstet Gynecol 2001; 17: 527-528.

[14]- Croitoru DP, Aaron LE, Laberge JM, Neilson IR, Guttman FM: Management of complex ovarian cysts presenting in the first year of life. J Pediatr Surg 1991; 26: 1366-1368.

[15]- Marshall JR: Ovarian enlargement in the first year of life: review of 45 cases. Ann Surg 1965; 161: 372-377.

[16]- Gohar J, Segal D, Hershkovitz R, Mazor M: Changing sonographic features of fetal ovarian cysts during pregnancy and the neonatal period. Arch Gynecol Obstet 1999; 263:82-83. 
Figure 1
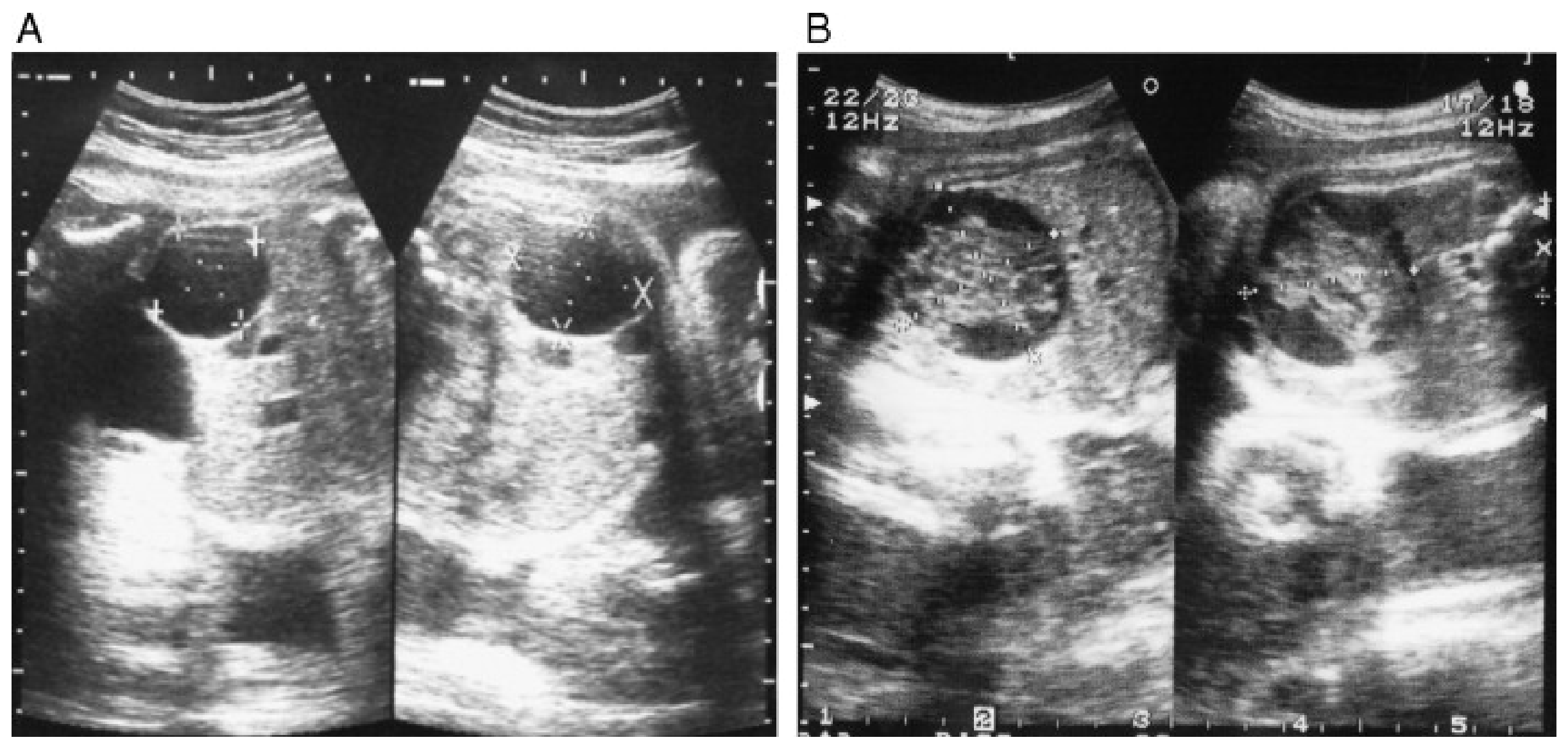
Table 1. Perinatal appearances of fetal ovarian cysts in relation to prenatal ultrasound pattern and size

\begin{tabular}{|c|c|c|c|c|c|c|c|}
\hline Case & $\begin{array}{l}1^{\text {st }} \text { prenatal } \\
\text { scan }\end{array}$ & $\begin{array}{l}\text { Size at prenatal diagnosis } \\
(\mathrm{mm})\end{array}$ & $\begin{array}{l}\text { Last prenatal } \\
\text { scan }\end{array}$ & $\begin{array}{l}\text { Maximum diameter } \\
(\mathrm{mm})\end{array}$ & $\begin{array}{l}\text { Outcome in } \\
\text { pregnancy }\end{array}$ & $\begin{array}{l}\text { Postnatal } \\
\text { outcome }\end{array}$ & Pathology \\
\hline 1 & s & 45 & $\mathrm{~s}$ & 45 & Reduced & S.R. & \\
\hline 2 & s & 48 & s & 48 & Reduced & S.R. & \\
\hline 3 & s & 75 & s & 75 & Reduced & Surgery & Simple cyst \\
\hline 5 & s & 31 & s & 34 & S.R. & - & \\
\hline 6 & s & 23 & s & 23 & S.R. & - & \\
\hline 7 & s & 41 & s & 41 & Reduced & S.R. & \\
\hline 8 & s & 44 & s & 66 & Increased & S.R. & \\
\hline 9 & s & 50 & $\mathrm{C}$ & 56 & No change & Surgery & Simple cyst with necrosis, calcification and torsion \\
\hline 11 & s & 49 & $\mathrm{C}$ & 49 & No change & Surgery & Simple cyst with hemorrhage \\
\hline 12 & $\mathrm{C}$ & 32 & $\mathrm{C}$ & 32 & No change & Surgery & Simple cyst with torsion and adhesion between bowel and ovarian cyst \\
\hline 13 & C & 42 & C & 46 & No change & Surgery & Simple cyst with necrosis, calcification and torsion \\
\hline 14 & C & 46 & $\mathrm{C}$ & 51 & No change & Surgery & Simple cysts with calcification and hemorrhage \\
\hline 15 & C & 40 & C & 40 & No change & S.R. & \\
\hline 16 & $\mathrm{C}$ & 55 & $\mathrm{C}$ & 55 & Reduced & S.R. & \\
\hline
\end{tabular}

